\title{
¿Ha quedado obsoleta la política de la diferencia?: Una exploración y propuesta.
}

\section{Has politics of difference become obsolete?: An explanation and the proposal.}

\author{
Máriam Martínez RAMíRez \\ Universidad Autónoma de Madrid \\ mariam.martinez@uam.es
}

Recibido: 10.11.2010

Aprobado definitivamente: 03.06.2011

\section{RESUMEN}

¿Cómo debería pensarse la política de la diferencia bajo condiciones de desigualdad estructural y diversidad cultural? ¿Ayuda la política de la diferencia a reforzar o a solventar esas desigualdades estructurales? En contextos de exclusión y marginalización social que también afectan a cuestiones de identidad, se propone un sentido renovado del término diferencia atendiendo a la diferenciación social, para que los procesos, las estructuras y las instituciones democráticas sean efectivamente más inclusivas.

Palabras Clave: Política de la diferencia, justicia social, redistribución, reconocimiento.

\begin{abstract}
How should the term Politics of Difference be thought under circumstances of structural inequality and cultural diversity? Does the Politics of Difference help reinforce or solve those structural inequalities? In order to answer these questions this article suggests a new meaning for the term difference attending to social difference in contexts of social exclusion and marginalization so that democratic institutions, processes and structures can be effectively more inclusive.
\end{abstract}

KEY words: Politics of Difference, Social Justice, Redistribution, Recognition.

\section{SUMARIO}

Introducción. 1.Una panorámica general. 2.El objeto y el sujeto: Igualdad individual, diferencia grupal. 3. ¿Redistribución o reconocimiento? 4. La diferenciación de la diferencia: la problematización del término cultura. 5. Un análisis de la multiculturalidad desde otra política de la diferencia. Conclusiones 


\section{INTRODUCCIÓN}

La política de la diferencia se ha utilizado tradicionalmente para nombrar a un conjunto de teorías basadas en la política de la identidad, asociada con reivindicaciones de justicia que claman por el reconocimiento de la diferencia cultural. Este artículo toma partido en esa afirmación para cuestionarla. Con este objetivo se pone en tensión la identificación entre diferencia e identidad, al mismo tiempo que se denuncian los peligros del esencialismo que la retórica de la identidad grupal normalmente plantea. Tras una exploración crítica, el artículo, sin embargo, refrenda la política de la diferencia como ideal democrático porque entiende que puede fomentar la inclusión dentro de la pluralidad que encierran nuestras sociedades. Para ello se proponen algunas revisiones del término "política de la diferencia" y un enfoque alternativo centrado en injusticias que surgen de diferencias estructurales como la posición social, la división del trabajo, la socialización de capacidades y los procesos de normalización que construyen qué es lo normal y qué lo desviado de una manera excluyente y opresora para determinados segmentos sociales.

El entendimiento de la diferencia como diferencia estructural se basa en una interpretación de la noción de justicia social que Iris Marion Young articula desde la teoría crítica (Young, 2000) . En su modelo de justicia social se argumenta a favor de una política que atienda a las diferencias sociales en los espacios de representación, comunicación y de la sociedad civil. Bajo las circunstancias de esa diferenciación estructural y cultural en la que viven nuestras sociedades, se interpreta esta política de la diferencia llevándola finalmente a casos concretos que ofrezcan respuestas alternativas y un tratamiento académico diferente al que tradicionalmente se viene dando a ciertos problemas que nacen de conflictos étnicos y religiosos, pero que esconden, como se verá, otros trasfondos.

\section{UNA PANORÁMICA GENERAL}

La discusión sobre la política de la diferencia y la identidad fue central para las reivindicaciones feministas, anti-racistas, colectivos de gays, minorías nacionales o étnicas, e incluso movimientos por la autonomía lingüística durante la década de los 80 , a partir de una denuncia centrada en desigualdades de género, raza, sexualidad, autonomía cultural y libertad religiosa o de culto. La forma de entender la diferencia entre estos grupos pronto se fue diversificando respecto de la versión estrictamente multiculturalista de la diferencia. ${ }^{1}$ En el contexto de resurgimiento de minorías nacionales y étnicas, las diferencias de nacionalidad, etnicidad y religión abanderaron lo que en ese momento comprendió el "paradigma del reconocimiento". La literatura producida en relación al tema había comenzado con el emblemático ensayo de Charles Taylor titulado Multiculturalism and the Politics of Recognition en 1992. En este ensayo, Taylor indagaba sobre las reivindicaciones de esos movimientos centrándolos en el reconocimiento de sus identidades específicas. Por eso sostenía:

Nuestra identidad está parcialmente modelada por el reconocimiento o por su ausencia, con frecuencia por el reconocimiento distorsionado de los otros, por lo que una persona o un grupo de personales pueden sufrir un daño real, una distorsión real, si la gente o la sociedad que está a su alrededor les devuelve un reflejo de sí mismos limitante, degradante o despectivo. (Taylor, 1992: 25) .

Sabemos que Taylor toma el término "reconocimiento" de La fenomenología del espiritu (1807) de Hegel renovándolo de una manera prodigiosa, y convirtiéndolo en el concepto más relevante de los autores insertos en este paradigma de la diferencia cultural. El trabajo de Taylor pronto recibiría un tratamiento mucho más sistemático en obras de Kymlicka como la magistral Multicultural Citizenship (1995), o las reflexiones de Axel Honneth en The Struggle for Recognition (1996) . La discusión política parece centrarse entonces en problemas ori-

\footnotetext{
${ }^{1}$ Sigo a Rafael del Águila en relación a su entendimiento del multiculturalismo como la "convivencia de entidades culturales más o menos cerradas y estables, donde el ámbito de encuentro en el que interactúan es, por así decirlo, "exterior", esto es, a la manera de "bolas de billar". Véase Del Águila (2008) : 464.
} 
ginados fundamentalmente por la diversidad cultural como nota distintiva de las sociedades modernas. Las sociedades reconocen ese pluralismo a partir de la visibilización de una pluralidad de grupos sociales que comprenden, como decimos, el espectro étnico, cultural, religioso y social.

En ese momento, el concepto de reconocimiento llegó a abarcar tanto que casi acabó perdiendo toda precisión para definir prácticamente nada. Pronto vendrían a mezclarse las reivindicaciones de un sinfín de movimientos dispares cuyas luchas comprenderían desde las apelaciones de justicia social de mujeres, hasta los nacionalismos periféricos, incluyendo las luchas culturales llamadas "subalternas" que competían por recursos y símbolos. Las reivindicaciones de todos estos grupos, ciertamente dieron lugar a muchas situaciones conflictivas. Por ejemplo, decidir la clase de educación que se daría en las aulas, o en qué lengua; el reconocimiento de determinados derechos especiales, la visibilización y plasmación legal de ciertas realidades sociales como el matrimonio homosexual; las cuestiones sobre qué tradiciones se podrían celebrar públicamente y cuáles, por el contrario, chocaban con los principios neutrales del Estado etc. Todas esas situaciones de diversidad ayudan de alguna manera a entender los conflictos que vienen produciéndose recientemente, a pesar de que algunos parecen más propios de otras épocas que del momento actual.

A grandes rasgos, puede decirse que este foco de atención surgido a partir de esa diversidad y pluralismo social ha generado tanto debate porque ha contestado de manera profunda algunos pilares del liberalismo. Ha puesto de manifiesto las amplias dificultades que surgen cuando intentamos pensar ciertos problemas bajo principios liberales. Han explicitado, en suma, los inconvenientes del pensamiento liberal para dar adecuadas respuestas a esos desafíos que han cobrado más visibilidad o relevancia en los últimos tiempos. En general puede decirse que la filosofía política contemporánea se haya inmersa en ese debate contra los límites del liberalismo. Y que ese debate se libra desde muchos frentes; desde comunitaristas, multiculturalistas y ecologistas, hasta republicanos y deliberativos. Para ciertos autores incluso, el debate más "productivo y animado" se desarrolla en el interior de la filosofía feminista (Wolff: 2001, 219). Partimos por tanto, de que el interés y la importancia del debate viene esencialmente del hecho de que estas corrientes cuestionan o problematizan los límites del liberalismo.

Es ampliamente asumido que la aparición del liberalismo supone por primera vez en la historia hablar de individuos y de igualdad entre esos individuos. $^{2}$ El sujeto político del liberalismo es el individuo, y el objeto la igualdad. Por el contrario, para todas estas teorías que hablan del reconocimiento y que centran el foco del debate en la diversidad cultural y social, el sujeto político deja de ser el individuo, y en su lugar se habla de grupos sociales, mientras que el objeto es, indudablemente, antes que la igualdad, la diferencia (a veces como fin en si mismo, a veces como medio para alcanzar la igualdad sustantiva). En cualquier caso, hablar de diferencia ha supuesto contestar la noción de igualdad como igual tratamiento y preguntarse algo tan obvio como “¿igualdad de qué?” (Phillips, 2004) y (Sen, 2009)

Debido a esas críticas formuladas contra el liberalismo, son frecuentes las asociaciones inconscientes entre diversas corrientes de pensamiento, que sin embargo, merecen matización y diferenciación. Por ejemplo, entre comunitaristas y multiculturalistas, o entre multiculturalistas y aquellos que hablan de la política de la diferencia. Pero, ¿realmente podrían comprenderse todas las apelaciones de estos movimientos dentro de la palabra "reconocimiento"? ¿Cómo deberían operar las políticas públicas en relación a ese término? ¿Es la política de la identidad lo mismo que la política del reconocimiento? ¿Qué supuso su aparición dentro del marco general de la teoría política donde Rawls había hecho un trabajo preeminente? ¿Qué debe implicar el término igualdad en relación con issues de justicia? ¿Qué es lo que debe distribuirse de forma igualitaria; las oportunidades, o los resultados?

\footnotetext{
${ }^{2}$ Asumo la posición del individualismo liberal clásico, y el supuesto de Locke según el cual los seres humanos son libres, iguales e independientes por naturaleza. Asumo también que con el presupuesto de que la sociedad es anterior a los individuos, el comunitarismo opta por una posición diametralmente opuesta a ese liberalismo individualista para el que el individuo es anterior a la sociedad.
} 


\section{EL OBJETO Y EL SUJETO: IGUALDAD INDIVIDUAL, DIFERENCIA GRUPAL.}

Puede decirse que el principio de igualdad constituye si no la más importante aportación de la tradición ilustrada liberal, al menos, una de las principales de ella. El logro ilustrado consistió en la posibilidad, por primera vez en la historia, de aplicar las mismas leyes y para todos. Con independencia de las diferencias individuales de cada cual, en relación a la riqueza o el estatus, se afirma que hay una consideración igualitaria de todas las personas en términos políticos dentro de la esfera pública. La radicalización de esta premisa la llevaría a cabo sin duda Kant, al afirmar que todas las personas tendrían el mismo valor moral y serían consideradas como fines absolutos.

Sin embargo, este logro supuso una consideración adicional. Implícitamente se estaba otorgando una "identidad igualitaria" (Young, 1996:102) a todas las personas por el hecho de ser ciudadanos. Una identidad de la igualdad definida en oposición a lo particular de cada ciudadano. Por este motivo algunos autores han señalado que a pesar de que tal concepto de ciudadanía pretendió la misma aplicación de derechos, deberes, de normas y leyes para todos, siguieron existiendo grupos de personas que se veían excluidos y marginados socialmente, sintiéndose de alguna manera, como "ciudadanos de segunda". Es cierto que esto podría tener una explicación marxista. Las actividades sociales que determinan el estatus social suelen ser oligárquicas, y además, los ciudadanos no tienen apenas control sobre la vida económica. Sin embargo, el análisis materialista de Marx no profundiza en los procesos simbólicos de la cultura en la creación de esos "ciudadanos de segunda". La formulación de la noción de ciudadanía entendida como generalidad y asimilación, daba lugar a la exclusión de aquellos que por sus diferencias culturales, valores, estilos de vida etc. no comulgaban con la definición de una categoría que había sido configurada por la clase burguesa privilegiada de hombres blancos de una determinada sociedad, en un determinado momento histórico.

En relación con la aspiración a la igualdad, algunos autores contemporáneos relevantes también han visto en la posición originaria de Rawls (1979) la eliminación de cualquier característica diferenciadora a través de lo que él denomina el velo de la ignorancia, y en la medida en que todas las personas parten de los mismos supuestos y de un punto de vista que es universal para llegar a lo que serán los principios de justicia. ${ }^{3}$ Numerosos teóricos de ese paradigma cultural han contestado que estas condiciones de imparcialidad e igualdad reducen la pluralidad de sujetos, con sus diversos puntos de vista. Y entienden además que un verdadero compromiso con la igualdad requiere atender antes que ignorar las diferencias.

Es aquí donde nos obligan a preguntarnos por las implicaciones del principio de igualdad en el contexto de democracias plurales que alejan cada vez más las respuestas de las instituciones de ciudadanía y soberanía hacia concepciones unitarias y homogéneas, sin que no obstante, pueda encontrarse un consenso claro dentro de las diversas corrientes. Mientras que Rawls sitúa esta igualdad en la distribución equitativa de los principales bienes sociales, otros autores como Sen hablan de una igualdad referida a la distribución de capacidades de los ciudadanos de una sociedad, en la que todos ellos reúnan las condiciones para ejercer ciertas capacidades como por ejemplo, el voto. Tal y como Sen lo diseña, el enfoque de las capacidades no centra la distribución en lo que representa el derecho al voto en sí, sino en garantizar la capacidad para ejercerlo, lo cual implica reconocer cosas tan variadas como el igual acceso a la educación, medios de información institucionales y alternativos, e incluso la facilitación del acceso a medios de transporte para ejercerlo. En la misma línea Martha Nussbaum (2002) pone de manifiesto que factores tales como la vulnerabilidad a la violencia física y simbólica de que son víctimas las mujeres representa un grave obstáculo en el desarrollo de las capacidades humanas, tanto cognitivas, como emocionales, e incluso imaginativas. Estos obstáculos, según Nussbaum, impiden el proceso de desarrollo de las capacidades de las mujeres en igualdad de condiciones que los hombres, pese a tener ganarantizados los mismos derechos que los varones (Nussbaum, 2006: 96-103) .

\footnotetext{
${ }^{3}$ Piénsese por ejemplo, en el "embate comunitarista" al "liberalismo igualitario" de Rawls, llevado a cabo por autores como Taylor (1985), Sandel (1982), Walzer (1983) o MacIntyre (1981) .
} 
En línea con el debate en torno a la igualdad, Dworkin ha distinguido entre la igualdad distributiva y la igualdad política, definiéndolas respectivamente como el derecho a acceder en igualdad de condiciones a las mismas fuentes y recursos (igualdad en términos de bienestar) por un lado, y el derecho, por otro lado, a ser tratados todos como iguales (Dworkin, 2000:11) . La interpretación de algunas de las ideas de Sen arriba expuestas permiten sin embargo formular una crítica contra Dworkin porque hacen entender que la igualdad de acceso a recursos carece de relevancia si no hay una igualación en capacidades. Por otro lado, y formulando una segunda crítica contra Dworkin, es casi irrefutable el hecho de que la consideración de la igualdad como igual tratamiento de todas las personas ha generado hasta ahora injusticias estructurales importantes para determinados individuos por el hecho de ser mujeres, homosexuales o de pertenecer a determinadas minorías raciales. Hay razones para creer que la política de pedir derechos iguales para todos no ha sido suficiente para satisfacer el ideal de igualdad. La aparición de las controvertidas políticas de acción afirmativa vienen justificadas en gran parte por este hecho. Y han sido contestadas desde el liberalismo precisamente porque, a través de las mismas, se trata a las personas como miembros de grupos antes que como individuos. Se entiende que el hecho de que alguien sea negro o blanco, hombre o mujer, debería ser irrelevante para determinar el trato que recibe. Se dice, en suma, que el tratamiento debe estar determinado por los méritos individuales y no por la pertenencia a un determinado grupo social.

Desde el paradigma cultural se ha argumentado que equiparar igualdad con igual tratamiento e igual distribución de derechos supone ignorar las profundas diferencias que existen en las posiciones sociales, división del trabajo, socialización de capacidades, normalización de estándares y formas de vida que continúan poniendo en desventaja a los grupos históricamente oprimidos. En otras palabras, "las leyes pueden estar libres de un defecto sin que la sociedad quede libre de ese defecto" (Wolff, 2001:220) . Es decir, que la igualdad de derechos no impide que siga habiendo formas sutiles de discriminación. Esas formas de discriminación se padecen normalmente por pertenecer a determinados colectivos sociales. Por estos motivos, para autoras como Young (2007), un compromiso sustancial con la igualdad debe requerir atender especialmente más que ignorar estas diferencias grupales. ¿Pero qué sentido tiene esta afirmación?

Es posible que Tilly la conteste cuando afirma que hasta el momento, el compromiso con una igualdad sustancial en la aplicación de normas y principios ha producido lo que él denomina una "durable inequality" (Tilly, 1999:193-229), es decir, la existencia de procesos estructurales donde la gente produce y mantiene ventajas para ella misma, y desventajas para otros, referidas por ejemplo, al acceso a determinadas fuentes de poder, toma de decisiones, mayor autonomía, posiciones sociales de prestigio, que a su vez, van relacionadas siempre con desigualdades económicas o materiales. En este sentido, se sostiene que ciertas desigualdades sólo pueden salvarse prestando especial interés a las diferencias. Esto es, que atendiendo a principios de justicia social, a veces es necesario discriminar. Y que palabras como "acción afirmativa" funcionan como eufemismos dentro de imaginarios políticos donde no es posible hablar de discriminación ni siquiera en términos positivos, porque no se cuestiona el hecho de que el valor máximo que ha de protegerse sea el de la no discriminación, o el de que todas las discriminaciones sean injustas.

Esos procedimientos preferenciales de promoción se han realizado allí donde las instituciones han encontrado discriminación intencional en el pasado (Friedman, 1985) . Esa discriminación intencional histórica se hizo específicamente sobre gente marcada por el género o por la raza. El reconocimiento de la diferencia aquí no se hacía como algo intrínsecamente bueno. El reconocimiento de la diferencia funcionaba más bien para formular una apelación de justicia, esto es, para reparar una injusticia. Tal apelación de justicia se articulaba sobre la base de adscripción a un colectivo social históricamente discriminado y marcado como desviado por esa diferencia. Esto conducía irremediablemente, según interpreto, a la contestación del sujeto político del liberalismo.

Como hemos dicho, parece que estas desigualdades afectan a individuos por el hecho de pertenecer a grupos sociales. Pero al mismo tiempo, buena parte del pensamiento liberal considera incorrecto tratar a las personas como miembros de grupos antes que como individuos. ¿Es posible, por ejemplo, entender las políticas de acción afirmativa como una ex- 
tensión de la idea de igualdad de oportunidades? En relación a esa cuestión otra pregunta pertinente que cabe hacerse al hablar de igualdad es ¿igualdad para quiénes? O más bien; ¿es compatible con el liberalismo reconocer derechos específicos sobre la base de afiliaciones grupales sociales y culturales? ¿Qué desafíos presentan estas políticas para el principio del mérito?

En los debates políticos surgidos durante los años 80, especialmente en Estados Unidos, muchas de las cuestiones dadas fueron formuladas a partir de políticas afirmativas dirigidas a miembros de grupos discriminados históricamente por su pertenencia a un determinado sexo, etnia o raza. Tanto las políticas públicas encaminadas a solventar tales cuestiones de justicia social, como los términos en los que se formulaban los debates, se articulaban en torno a un vocabulario donde se dividía a la gente por categorías de género, edad, religión, tendencia sexual etc. Era frecuente además, la intersección de esos niveles de análisis a partir de los cuales se dotaba a la gente de muchas identidades (mujer, blanca, heterosexual etc.) .

En el marco de todas esas intersecciones identitarias es donde los autores del paradigma de la diferencia o el reconocimiento han visto con más facilidad encontrar e identificar desigualdades estructurales que afectan a procesos sociales y relaciones institucionales, antes que a injusticias que se dan bajo relaciones estrictamente individuales (Young, 2007. Taylor, 1992. Phillips, 2004) . Dworkin por el contrario, ofrece su teoría como moral y metodológicamente individualista, en la medida en que entiende que el principio de igualdad es una cuestión de "derecho individual antes que de una posición grupal" (2004) . Sin embargo, el lenguaje de los grupos sociales frente al de individuos según Hartney, genera confusiones porque "nos distrae respecto del hecho de que la última razón para la necesidad de proteger colectividades es su contribución al bienestar de los individuos" (Hartney, 1995:204) . El argumento que da Hartney, según interpreto, podría valer para probar el argumento contrario, esto es, que el reconocimiento de derechos colectivos sea algo bueno precisamente porque abre las puertas al desarrollo y bienestar individual, y en ese sentido, no debería ser incompatible con el liberalismo. Así nos lo hace saber Phillips, al poner de manifiesto, en primer lugar, que la igualdad de resultados debe relacionarse es- trechamente con la igualdad de oportunidades, que esa igualdad de oportunidades y de resultados no depende tanto de la elección y responsabilidad individual como del marco social e institucional donde los sujetos deben tomar esas decisiones, y que es propio de las categorías de grupos sociales evaluar esa igualdad de oportunidades y de resultados ( $\mathrm{Ph}$ llips, 2004:6-9) .

La conexión que hace Phillips entre oportunidades y resultados supone cuestionar implícitamente el principio del mérito, como principio regulador de posiciones sociales. No obstante, resulta un tanto ingenuo pensar que se puede prescindir de un principio regulador que distribuya posiciones laborales, y que no lo haga midiendo de alguna forma, la competencia individual de las personas. Una extensa bibliografía del ámbito de la teoría social presupone con frecuencia que para aplicar el principio del mérito debe ser posible identificar y evaluar la actuación individual utilizando criterios que sean neutrales (Nielsen, 1985:104-112) . Otros autores opinan que la neutralidad no existe porque "la mayoría de los criterios de evaluación usados en nuestra sociedad, incluyendo las referencias educativas y las evaluaciones estandarizadas, tienen contenido normativo y cultural" (Young, 1990:325) . Como consecuencia, la cuestión central de la justicia en este ámbito debería centrarse en quién decide cuáles son las aptitudes adecuadas para una determinada posición, cómo se valoran dichas aptitudes, y cómo se comprueba si los individuos las tienen. Tanto Young como Phillips se postulan a favor de la discriminación positiva, como lo hacen la mayoría de los autores que venimos catalogando dentro del paradigma del reconocimiento y política de la diferencia, frente al liberalismo igualitario de autores como Nielsen o Brian Barry para quien, desde una postura muy crítica con las políticas de la diferencia, defienden que las posiciones laborales con mayor rango de autoridad deben ser ocupadas por aquellos individuos que demuestran el mayor grado de excelencia que se espera para desarrollar esa clase de trabajos. De esta manera, Barry asume que un principio estándar de mérito, una vez garantizada la igualdad de oportunidades, debe rechazar todo sistema que reserva explícitamente determinadas posiciones en conformidad con características que tienen que ver con el género, la clase social o el grupo étnico (Barry, 2001:98-103) . 
Las distancias en los postulados y las argumentaciones del liberalismo igualitario frente a la política de la diferencia han demostrado ser lo suficientemente fuertes como para no haber encontrado consensos unitarios ni siquiera en torno a los valores que debe preservar cualquier concepción de justicia. Esta situación queda descrita por Fraser (1997) en un libro que sigue siendo un referente actual para cualquier debate sobre la teoría de la justicia social. ${ }^{4}$ La obra de Fraser desde las primeras páginas, sitúa el foco de todas divergencias teóricas postsocialistas en torno a la dicotomía entre redistribución y reconocimiento. Al análisis de Fraser pasamos ahora.

\section{3. ¿REDISTRIBUCIÓN O RECONOCIMIENTO?}

En 1997, Nancy Fraser escribe un libro llamado Iustitia Interrupta donde habla de la condición postsocialista como aquella que refiere a la paradoja de un "desplazamiento en la gramática de las de reivindicaciones políticas" que convive con la ausencia de una alternativa emancipatoria creíble (Fraser, 1997:4) .

Lo que ha fracasado (...) no es solamente un conjunto de (arcaicos) acuerdos institucionales que existieron realmente, sino también la creencia en el principal ideal que inspiró las luchas por la transformación social durante el último siglo y medio. (...) Al menos por el momento, no ha surgido una nueva visión progresista omnicomprensiva que ocupe el lugar del socialismo. Las propuestas por elevar la "democracia radical" y el "multiculturalismo" a esa categoría expresan el deseo de algunos por encontrarla. No obstante, en mi opinión, carecen de poder de convicción porque ponen entre paréntesis el problema de la economía política. Lo mismo vale para las aún más anémicas nociones de "liberalismo político" y "comunitarismo" (Fraser, 1997:4) .

Normalmente, sostiene Fraser, las reinvindicaciones por la redistribución (centradas en injusticias definidas como socioeconómicas) y por el reconocimiento (centradas en injusticias definidas como culturales) (Fraser, 1997:6), junto con las luchas que sendas concepciones impulsan, han constituido dos paradigmas de justicia diseñados casi de manera excluyente. Y en los últimos años, asistimos a un fenómeno en el que las reivindicaciones por el reconocimiento de la diferencia arraigadas en injusticias de tipo cultural y simbólico, han ido arrinconando progresivamente a las reivindicaciones por la igualdad social, surgidas de las estructuras económicas. Este cambio de paradigma viene protagonizado por un conjunto de grupos sociales que plantean su lucha social de emancipación frente a unos cánones de dominación que dejan de trazarse en términos marxistas, para dar prioridad a otro imaginario político expresado a través de las nociones de identidad y diferencia. Según Fraser, la condición "postsocialista", surgida tras la caída del muro de Berlín, se caracteriza en suma, por un descalabro de la izquierda ortodoxa "que incluye una tensión un poco parecida a la de la mañana siguiente", a la sensación de "un volar sin instrucciones" y a un "agotamiento de las energías utópicas de la izquierda", que la conduce a buscar su ideal emancipatorio en la política de la identidad. Esa ausencia de alternativa no justifica, sin embargo, la "afirmación hueca" de Francis Fukuyama sobre el fin de la historia. Más bien refleja, señala Fraser, la complacencia acrítica con el estatus quo.

Ese giro hacia la política de la diferencia se hace cuando un conjunto de movimientos sociales relacionados con la política de izquierdas no ven satisfechas sus aspiraciones dentro de las concepciones tradicionales de justicia que no atienden a cuestiones de imperialismo cultural, por ejemplo. De manera que se pone de manifiesto que existen fuentes de desigualdad estructural que van más allá de una noción de justicia distributiva, pero que tampoco pueden englobarse y abarcarse propiamente a través de la lógica de la política del reconocimiento. La división del trabajo que lleva implícita la definición de tareas y cualificaciones en el seno de ocupaciones dadas, el poder de toma de decisiones sobre la inversión pública y privada, y el monopolio sobre cánones de representación e interpretación,

\footnotetext{
${ }^{4}$ Véase por ejemplo, Barnes, M., Newman, J., Sullivan, H. en Power, Participation and Political Renewal: Cases Studies in public participation, The Policy Press, 2007, pp. 33-53. O Benhabib, S., The Claims of Culture, Princeton University Press, 2002, pp.49-82.

${ }^{5}$ Fraser recurre al texto de Habermas "The New Obscurity and the Exhaustion of Utopian Energies", en Observations on the Spiritual Situation of the Age, Cambridge, MIT Press, 1984.
} 
podrían ser cuestiones de justicia social que integran esas apelaciones de los movimientos sociales ligados con la nueva política de izquierdas (Young, 1997b:151) . De alguna forma, estos movimientos estaban demandando salir del paradigma meramente distributivo, al mismo tiempo que sentían que sus aspiraciones no podían verse satisfechas a partir de un concepto de justicia centrado exclusivamente en el reconocimiento cultural.

Fraser señala que el coste del surgimiento de la política de la identidad se traduce en un cambio gramatical (ya no se habla de clase social, sino de grupo cultural), y en una transformación más profunda referida al imaginario político "especialmente en los términos en los que se concibe la justicia" (Fraser, 1997:5) ; porque la condición postsocialista define ahora al paradigma cultural, en oposición al paradigma distributivo-socialista, en una "versión totalizante de la diferencia" que todavía hoy no responde cabalmente a las exigencias de economía política. Fraser proyectará casi todos los esfuerzos de su obra, desde principios de los años 90 a nuestros días, ${ }^{6}$ a buscar la fórmula teórica que favorezca la integración de ambos paradigmas, y situará el ejemplo paradigmático de esa necesidad en el caso del género y de la raza. Según la autora, la desigualdad de género por ejemplo, es correlativa al fenómeno de la feminización de la pobreza, lo cual requiere medidas de justicia distributiva. Pero también implica la necesidad de desestabilizar un imaginario colectivo que asume que las mujeres deben emplear sus energías en el desempeño de determinados trabajos más "aptos" para ellas, lo que requiere medidas de justicia cultural o simbólica.

El panorama descrito por Fraser, quedaría expuesto gráficamente de la siguiente manera:

\footnotetext{
${ }^{6}$ Es importante señalar que en ese modelo de justicia bidimensional, Fraser ha incorporado el nexo justicia-democracia, tratando la representación como la tercera dimensión de la justicia. Véase Fraser (2008).
} 


\section{JUSTICIA SOCIAL}

\section{JUSTICIA CULTURAL}

IGUALDAD

Afecta

Afecta

INDIVIDUOS

AUTORES REPRESENTATIVOS

(1) Rawls

(] Dworkin

PARADIGMA

REDISTRIBUTIVO

Propone

IGUALDAD COMO EQUIDAD

Abarca

\section{INJUSTICIA SOCIAL}

Colectividad social definida por la clase social.

\section{ESTRUCTURA TEÓRICA \\ $\square$ Universalidad \\ (.) Generalidad \\ $\square$ Abstracción. \\ $\square \quad$ Teoría social positivista}

TRANSFORMACIÓN

CULTURAL O SIMBOLICA

Abarca

AUTORES REPRESENTATIVOS

Kymlicka

ㄱ Taylor

Propone

INJUSTICIA CULTURAL

Colectividad social de homosexuales y lesbianas.

ESTRUCTURA TEÓRICA

Contextual

(1) Pragmática

○ Teoría crítica 
Al sostener que existen dos paradigmas de justicia, ${ }^{7}$ Fraser trata de explicar la dinámica de las luchas sociales en nuestras democracias contemporáneas. Clasificándolas de esta manera, la autora pretende dar cabida a las reivindicaciones por la igualdad social y económica, y por el reconocimiento de la diferencia. Como puede verse en el esquema, su tesis es que el género y la raza constituyen colectividades bivalentes que atraviesan el espectro de la redistribución y del reconocimiento. Para eliminar la discriminación de género y de racismo, se requiere no sólo una transformación y reestructuración del nivel de las relaciones socioeconómicas, sino también, del nivel del espectro del reconocimiento. Los casos puros que afectan exclusivamente a uno de los paradigmas serían el de los colectivos de gays y lesbianas, afectados, según Fraser, por una injusticia de tipo cultural y simbólico, y el de la clase social definida en términos marxistas, a cuyas reivindicaciones se dirige, según ella, $A$ Theory of Justice de John Rawls.

Al contrario de lo que podría parecer, dice Fraser, tampoco podría afirmarse que el propio Rawls elabora una teoría omnicompresiva de justicia porque sustrae las problemáticas culturales del alcance mismo de justicia. Ciertamente, en Political Liberalism, Rawls articula una ingeniosa propuesta que combina elementos kantianos y utilitaristas para formular un "consenso entrecruzado" (overlapping consensus) que sea respaldado por la mayoría de los ciudadanos y que trate de lidiar con la gran variedad de doctrinas existentes en nuestras sociedades pluralistas. ${ }^{8}$ Este "consenso entrecruzado" responde fundamentalmente a las críticas comunitaristas hechas al individualismo liberal subyacente en su teoría, ${ }^{9}$ y por ello, representa el esfuerzo del pensador por integrar dentro de su teoría de la justicia las diferencias de valores y culturas existentes. La idea de consenso entrecruzado, pues, sugiere la necesidad de un conjunto de principios básicos para gobernar la vida política, que se construye a partir del común acuerdo surgido de ese entrecruzamiento de "doctrinas comprehensivas razonables, (no irrazonables o irracionales) " (Rawls, 1996:76).$^{10}$ El concepto de consenso por superposición, pues, sería "el acuerdo sobre las concepciones políticas centrales al que acceden personas que se adscriben a doctrinas o concepciones del bien distintas o incluso opuestas" (Vallespín, 1996:18) .

Sin embargo, la idea de un consenso generalizado moral sobre creencias acerca de la vida buena, sobre cómo la gente debería actuar en la sociedad, sobre cómo la sociedad debería estar ordenada, es quizás demasiado vaga y general como concepto para abarcar debidamente la pluralidad de nuestras sociedades modernas. Los conflictos que surgen en

\footnotetext{
${ }^{7}$ Fraser (1997) : 5. La misma tesis puede encontrarse en Fraser (1997a)

${ }^{8}$ Hay en efecto, un cambio significativo entre A Theory of Justice (1979) y Political Liberalism (1996) derivado de los términos en los que cada trabajo sitúa el conflicto político, a pesar de que Rawls declara que el cuerpo básico de las afirmaciones e ideas contenidas en $A$ Theory of Justice permanecen inalteradas en Political Liberalism. Puede hablarse en ese sentido de una evolución procedimental que le hace ir de un constructivismo moral a un constructivismo político. Una lectura cuidadosa de Political Liberalism podría dejar ver que Rawls desde las primeras páginas, está situando el problema primordial de una teoría de la justicia en factores derivados del pluralismo cultural. Así lo expresa, por ejemplo, cuando dice "Under the political and social conditions secured by basic rights and liberties of free institutions, a diversity of conflicting and irreconcilable, and reasonable, comprehensive doctrines will come about and persist if such diversity does not already obtain" en RAwLs (1996) :. La preocupación principal de Rawls aquí es cómo conseguir esa "cooperación social" dentro de un contexto social caracterizado por su pluralismo cultural, para que de entre todas las diversas concepciones del bien razonables existentes y correspondientes a esa diversidad cultural, ninguna se sienta oprimida o restringida. La idea del overlapping consensus surge para dar respuesta a este interrogante. A Theory of Justice, sin embargo, sitúa el conflicto político que ocasiona el problema primordial de una teoría de justicia en los intereses individuales por buscar y conseguir bienes sociales y recursos para que los individuos puedan realizar sus planes racionales de vida. Véase Rawls (1979) : 128. Political Liberalism focaliza más el conflicto político en la diversidad de creencias y concepciones de vida buena, posiblemente porque cambia la metodología. Ello a su vez, le hace abandonar la idea de un velo de la ignorancia que ponga entre paréntesis las diversas creencias y concepciones de vida buena existentes en sociedades plurales.

${ }^{9}$ En relación al "embate comunitarista" contra lo que se articula como una concepción demasiado atomista de sujeto dentro de $A$ Theory of Justice de John Rawls, véanse especialmente las críticas formuladas en las obras de Sandel (1982), Taylor (1979), Walzer (1983) y Macintyre (1981) .

${ }^{10}$ Efectivamente para Rawls, sólo aquellas doctrinas comprehensivas razonables formarán el consenso entrecruzado para llegar a los principios de justicia. Sugiero que la idea de que un valor cultural o creencia sobre la naturaleza de la realidad forme un sistema coherente racional, refleja un profundo sesgo occidental y tiende a privilegiar aquellos segmentos sociales cuya cultura se presta más a ser expresada precisamente en estos términos sistemáticos y racionales. 34 VALLESPÍN (1996) : 18
} 
sociedades pluralistas no se deben muchas veces al choque de creencias articuladas racional y sistemáticamente en doctrinas diversas, sino a prácticas, a menudo incomensurables, que resultan de la utilización de recursos, de diferentes formas de ver el mundo o diferentes maneras sustanciales de concebir al ser humano y sus pautas de actuación; de distintas maneras de concebir el tiempo, de distinguir las habilidades y valores importantes que se ve en la gente; de diferentes manifestaciones de expresión corporal, formas de dirigirse a la gente, maneras de hablar, distintos hábitos corporales, gustos etc. Ninguna de todas estas significaciones puede reducirse a cultura tal y como la expresa Rawls, y tal como la articula el paradigma del reconocimiento. La propuesta de Rawls ofrece una perspectiva sensible a la política de la diferencia debido a las pautas generales que el autor sistematiza "para tratar de teorizar una solución pluralista a los retos políticos planteados por (la) diferencia" (Vallespín, 1996:183) . En este artículo, sin embargo, se propone otro entendimiento de la diferencia que comienza por la deconstrucción misma del término cultura desde otros ejes sociales estructurales basados en la división social del trabajo, la sexualidad o la normatividad corporal.

Como ya hemos señalado, la expresión "política del reconocimiento" es introducida por Charles Taylor a propósito de su ensayo Multiculturalism and politics of recognition ${ }^{11}$ para referirse a la formación intersubjetiva de identidades individuales, y a la importancia del reconocimiento como interacción recíproca de respeto para el desarrollo de una concepción positiva del yo. Según Fraser, la política de la identidad supone un paso adelante en las aspiraciones por ampliar la noción de justicia, porque da cuenta de la importancia de patrones y roles sociales de representación, interpretación y comunicación en el desarrollo individual, y de las injurias de irrespeto y subvaloración producidas por imágenes tendenciosas y asociaciones simbólicas. A diferencia de Taylor, Fraser parece otorgar un contenido más empírico al concepto de reconocimiento. Mientras reconocimiento en Taylor implica más bien autoafirmación, lucha por la autonomía cultural y la tolerancia para la libertad religiosa, en Fraser, por el contrario, supone una acción afirmativa encaminada a desestabilizar ese tipo de patrones culturales de interpretación, comunicación y representación. En este sentido, es en el que afirmo que la expresión "reconocimiento" es demasiado amplia como para comprender o analizar determinadas demandas de grupos sociales que sin embargo, sí que coinciden en poner en cuestión algunos presupuestos básicos del liberalismo clásico como la consideración política del grupo social, frente al individuo o la aplicación de los mismos principios de evaluación y distribución a todas las personas. Veamos más detenidamente cómo se establece esta diferenciación dentro de la diferencia.

\section{LA DIFERENCIACIÓN DE LA DIFERENCIA: LA PROBLEMATIZACIÓN DEL TÉRMINO CULTURA.}

El surgimiento de la cultura como un lugar de controversia política es uno de los rasgos más paradigmáticos de la situación actual. Los debates sobre planes de estudio multiculturales, sobre conflictos religiosos como el caso del velo islámico, la defensa basada en criterios culturales dentro de la jurisprudencia criminal en los Estados Unidos etc. han convertido la palabra cultura en un instrumento de diferenciación y reivindicación social.

Como ya se ha dicho, a partir de finales de los años 80 , numerosos autores también comienzan a reparar en un cambio teórico notorio, centrado en las ideas y reivindicaciones de reconocimiento cultural. En relación a ello, Seyla Benhabib afirma:

Lo que resulta novedoso es que los grupos que actualmente se constituyen en torno de dichos indicadores identitarios exigen el reconocimiento legal y la distribución de los recursos del Estado y de sus organismos para preservar y proteger sus especificidades culturales. La política identitaria involucra al Estado en guerras culturales. Por consiguiente, el propio concepto de cultura ha cambiado (Benhabib, 2006:22) . .

En la sugerente Culture \& Equality: An Egalitarian Critique of Multiculturalism (2001), Brian Barry diferencia entre los significados y usos de cultura

${ }^{11}$ Sigo la interpretación de Seyla Benhabib en (Benhabib, 2006:96) . 
dados por Kymlicka e Iris Young respectivamente. ${ }^{12}$ Huelga decir que el estudio y la identificación de los matices sustanciales que la palabra cultura juega en la obra de esos dos autores, no disuade a Barry del hecho de formular una crítica devastadora dirigida hacia aquellos autores que "politizan la cultura". ${ }^{13}$ En esa crítica se puede identificar con toda nitidez que dentro de la literatura política fundamental es posible diferenciar, como poco, dos modos de usar el término cultura.

La definición de cultura que cita Barry comentando la obra de Iris Young, hace referencia a símbolos, imágenes, comportamientos, etc. a través de las cuáles la gente experimenta y se comunica con otra gente. ${ }^{14}$ En este sentido, cultura puede entenderse como acción comunicativa, antes que como una entidad sustancial, cercada y coherente, distinguida de forma nítida de otras culturas. Esto es, cultura como "totalidad claramente delineable" (Benhabib, 2006:27) . Por otro lado, Kymlicka define el término "cultura societal" como "comunidad intergeneracional, definida más o menos institucionalmente, que ocupa un territorio dado y comparte una historia y lengua definida" (Kymlicka, 1995:18) . Entendida de esta forma sustantiva, Kymlicka nos habla de una política de la identidad que comprende el concepto de cultura como algo sustantivo, como un conjunto de significaciones compartidas referidas a una forma de vivir expresada por una comunidad, que quiere preservarse y preservar esa forma de ver la vida. En ese sentido, cultura se convierte en algo que la política de la identidad debe cuidar, mientras que en la acepción de Young, cultura es expresión comunicativa, recurso político para la acción y transformación, y también fuente de injusticia estructural. La versión de la política de la identidad que nos ofrece Kymlicka concuerda con una política de la diferencia destinada a grupos étnicos, nacionales o religiosos, mientras la segunda haría referencia a grupos sociales de identidad colectiva formados a partir del género, la clase social, la raza y las sexualidades "denostadas". Como ya hemos visto, según Fraser, la mayoría de estos movimientos sociales incorpora en sus luchas tanto aspectos por el reconocimiento, como por la distribución. En línea con esto, sus reivindicaciones entrarían a formar parte de la política de la diferencia no entendida como identidad, sino como recurso político.

Tanto Young, como Fraser, como la propia Benhabib, entienden que las reivindicaciones de esta política de la diferencia podrían computarse como cuestiones de justicia universal (Benhabib, 2006:13) . Para Young, sin embargo, las apelaciones de justicia desde la política de la diferencia deben dirigirse en primera instancia a injusticias relacionadas con una desventaja estructural asociada especialmente con el género, la clase, la posición racial o la habilidad, antes que como materias de diferencia cultural como tal. Autoras como Ann Phillips, Amy Gutmann o el propio Paul Kelly comparten esta idea (Phillips, 200;Gutmann, 2004; Kelly, 2002) .

Según Benhabib, a pesar de que "el imaginario político catapulta al primer plano del discurso político el tema de la identidad cultural, en el sentido más amplio del término", las reivindicaciones de esos movimientos sociales han sido sistematizadas de forma diversa por los diferentes autores: "luchas por el reconocimiento" según Taylor y Honneth, "movimientos por la diferencia", según Iris Young y William Connolly, y "movimientos por los derechos culturales y la ciudadanía multicultural", según Will Kymlicka (Benhabib, 2006:8) . La diferenciación dentro de este conjunto de autores podría quedar establecida, según interpreto, de la siguiente manera: política del reconocimiento (Taylor y Honneth) , política de la diferencia como desigualdad social (Young), y por último, política de la identidad o de la diferencia cultural (Kymlicka) .

De ninguna manera esta clasificación pretende agotar todas las posibilidades. Debe haber otras muchas, e incluso éstas podrían estar abiertas a una mayor profundización y explicación. Creo, sin embargo, que esta pequeña sistematización ayuda a entender parte del sentido del artículo, y especialmente, algunas de las propuestas contenidas en él. Una de las principales razones para distinguir teóricamente las distintas versiones de la política de la diferencia es la prioridad en relación a cuestiones de justicia

\footnotetext{
${ }^{12}$ Véase especialmente (Barry, 2001:92-102).

${ }^{13}$ En ese empeño por "politizar la cultura", Barry incluye a muchos otros autores como James Tully, Bhikhu Parekh, Nancy Fraser, etc. Algunas buenas réplicas a las tesis de Barry en este punto se pueden encontrar en (Benhabib, 2006:190-93) y en (Kelly, 2002:62) .

${ }^{14}$ Esa definición puede encontrarse en (Young, 1990:23).
} 
que establece cada una de ellas. Según se interpreta en este artículo, la diferencia como identidad suele centrarse en cuestiones sobre libertad para ejercer prácticas culturales y formas de expresión, mientras que la diferencia entendida como desigualdad estructural pretende dirigirse primeramente a injusticias causadas por las limitaciones en oportunidades y desarrollo de capacidades que alguna gente sufre como consecuencia de ejes sociales estructurales e institucionales. No obstante, es importante tener en cuenta que muy a menudo, la existencia de diferencias culturales provoca un agravamiento en las condiciones de desigualdad estructural, y que hay conflictos de índole cultural que no pueden desligarse de otra clase de factores estructurales en sentido de desigualdad de acceso a recursos económicos, políticos y sociales. El siguiente apartado lo dedicaremos a analizar en qué sentido la política de la diferencia como desigualdad social (Young) puede visibilizar otras cuestiones de justicia social que no se reducen estrictamente a la libertad de expresión cultural o identitaria. Esto es, en qué sentido "diferencia" llega a ser desigualdad estructural, antes que expresión cultural religiosa, nacional o étnica, y cómo sobre la base de conflictos aparentemente multiculturales, es posible detectar esa diferencia como desigualdad estructural que suele pasar desapercibida cuando se mira desde la sola óptica multiculturalista.

\section{UN ANÁLISIS DE LA MULTICULTURALIDAD DESDE OTRA POLÍTICA DE LA DIFERENCIA.}

La mayoría de los conflictos multiculturales producidos en el seno de democracias occidentales suelen aparecer como cuestiones relacionadas con prácticas culturales específicas: celebración de determinadas fiestas, uso público de símbolos, dispensa de otras prácticas hechas por la cultura mayoritaria o de acogida etc. El conflicto político en estos casos suele limitarse a aquel en el cual el grupo dominante debe encajar dentro de sus valores y principios las prácticas y las formas de expresión que son signo de identidad de otra cultura. Normalmente, la visibilización de estas cuestiones pasa por la discusión sobre procesos de acomodación y reconocimiento de la diversidad, y sólo por esto. Otros temas suelen pasar desapercibidos en relación a desigualdades estructurales que muchas veces se construyen sobre la base de esas diferencias culturales, o como consecuencia de una identificación del otro diferente perteneciente a una cultura marcada como desviada. En este artículo señalamos algunas de esas cuestiones que tienen que ver con:

i.Procesos de construcción de lo normal y lo desviado.

\section{ii. Procesos de invisibilización de otros conflictos.}

iii. Procesos de estigmatización, segregación y racialización.

\section{i. Procesos de construcción de lo normal y lo desviado.}

Partimos del concepto de normalización que Young elabora sobre la base de las ideas de Foucault y Michael Warner (1999) . La normalización ocurre, pues, cuando se construye la experiencia y las capacidades de algunos segmentos sociales en estándares contra los cuales son medidos y consecuentemente valorados como desviados o deficientes otros miembros de la sociedad (Young, 2006:96) . La normalización se produce cuando instituciones públicas y prácticas sociales generan una clase de expectativas sobre ciertos individuos para que muestren comportamientos o capacidades determinadas, asumidas como "normas", cuando éstos, por su diferencia, son completamente incapaces de ostentarlas, o lo hacen a costa de sufrir una experiencia vejatoria o denigrante. Desde esta perspectiva, existen procesos de normalización masculina, normalización racial o etnocéntrica, normalización sexual, y normalizaciones que afectan a gente con ciertas capacidades limitadas.

¿En qué medida estos procesos de normalización pueden influir sobre miembros de culturas étnicas minoritarias dentro de democracias occidentales? Es en relación al principio de tolerancia desde donde esta operación normalizadora puede identificarse, por lo general, con mayor cierta nitidez. En los debates sobre determinados temas, suele verse hasta qué punto se construye el punto de vista del "nosotros", discutiendo (normalmente, dentro de si mismo) hasta qué punto es posible acomodar o afirmar una cultura que por lo general, suele marcarse como desviada. 
La idea de tolerancia no se puede entender sin sus límites. Y sin embargo, con frecuencia son esos mismos límites los que definen el adentro y el afuera como lo normal y lo desviado, incluso cuando determinadas prácticas están condenadas por aquellos mismos a los que se les marca como desviados. Cuando se da opción a los "desviados" a expresar sus propios puntos de vista sobre determinadas cuestiones, lo hacen desde esa posición de "anormales" que en buena medida, ya predispone los términos y las conclusiones del debate.

\section{ii. Procesos de invisibilización de otros con- flictos.}

Hasta donde se ha tenido ocasión de ver en este artículo, parece ir de suyo afirmar que el término reconocimiento tiende a deslizarse entre diversos significados, dentro de los cuales, ninguno de ellos parece centrarse en sentido estricto, en cuestiones de estigmatización y exclusión social. En enfoques multiculturales, el término reconocimiento se invoca para apelar a la necesidad de reconocer estilos de vida, costumbres, tradiciones o cuestiones de autogobierno. Normalmente, dentro del marco teórico multicultural y de la política del reconocimiento, los debates que atañen a cuestiones de género (generalmente, la mayoría) son usados, antes que como fines en sí mismos, como medios para probar la eficacia y validez de las propias teorías y principios de tolerancia. Estos temas suelen presentarse como injusticias que sufren las mujeres, y acostumbran, según Young, a tener un gran efecto retórico para legitimar acciones políticas "ciertamente cínicas" como la invasión estadounidense de Afghanistan en 2001 (Young, 2007:111) .

En estos casos hablamos de la invisibilización de las cuestiones de género, porque lo que se debate son principios liberales más generales y abstracto sobre la libertad religiosa o el principio de tolerancia. ${ }^{15}$ De esta manera, se acostumbra a ocultar otras cuestiones estructurales de explotación, marginalización y subordinación que afectan directamente a las mujeres que son tratadas como objetos de esos debates multiculturales más genéricos.
¿Pero por qué se propone aquí la aproximación de estas problemáticas desde una diferencia como diferenciación estructural, antes que desde el reconocimiento de una diferencia entendida desde el marco multiculturalista? Se habla de política de la diferencia en estos casos, en primer lugar, porque el reconocimiento de esta diferencia ayuda a visibilizar directamente procesos de explotación, marginalización y subordinación que las afecta a ellas como grupo social definido por el género, antes que como miembros de sus comunidades étnicas. Como consecuencia se sostiene que antes de discutir por ejemplo, la pertinencia del uso público del velo, se debería hablar de otros factores estructurales como las condiciones de igualdad de oportunidades en el desarrollo de capacidades para una muchacha decida por si misma si experimenta la práctica del velo como expresión u opresión cultural. En línea con lo dicho anteriormente, se entiende que la estrategia política aquí es primero la de reconocer la diferencia sexual, esto es, que un grupo de personas sufren vejación porque pertenecen a un grupo o colectivo social, y seguidamente, que es necesario fomentar políticas públicas especiales y específicas para solventarlas. Según esto, ¿por qué se debería hablar como diferencia en sentido de desigualdad estructural, antes que de diferencia entendida como identidad? Porque desde el paradigma de la diferencia entendida como desigualdad estructural, se conceptualizan las cuestiones de género a partir de la óptica de un posicionamiento estructural que pone de manifiesto la vulnerabilidad que sufren las mujeres a causa de una dominación que atraviesa ejes estructurales como la división sexual del trabajo, la socialización de género y la normatividad sexual. Aquí las mujeres no reivindican la defensa de una identidad común, sino la visibilización de unos ejes estructurales que perpetúan una injusticia, y que requiere de la adopción de medidas específicas.

Estos ejes estructurales vertebran la mayoría de las sociedades occidentales. Por ello las reivindicaciones culturales, no deberían separarse de este otro tipo de reivindicaciones que afectan más directamente a mujeres porque hablan expresamente sobre patrones de socialización de género o de estructuras opresoras de división sexual del trabajo.

\footnotetext{
${ }^{15}$ Tomo la idea de Seyla Benhabib para analizar lo que para ella es una forma errónea de abordar los conflictos multiculturles. Véase (Benhabib, 2002: 21-22)
} 
iii. Procesos de estigmatización y segregación.

Otro de los ejes estructurales que suele pasar desapercibido dentro de las discusiones y enfoques multiculturales es aquel que hace referencia a procesos de socialización de modelos corporales que naturalizan y normalizan características corporales, frente a otros que aparecen como desviados o inferiores; a procesos en suma, de socialización de cuerpos, que sirven de excusas para producir y reproducir desigualdades estructurales porque debido a tales atributos físicos, se posiciona estructuralmente a alguna gente de forma abyecta.

Dentro de los enfoques multiculturales, con frecuencia se utiliza la palabra "etnicidad", que ha sustituido a la palabra "raza" (Young, 2007:115) . De esta manera, se distingue "raza" de "etnicidad" de acuerdo a un proceso que naturaliza en términos "epidérmicos"16 diferentes atributos corporales como son el color de la piel, el tipo de pelo o los rasgos faciales. En sociedades occidentales, el racismo suele ser el resultado de un proceso de jerarquización de cuerpos que organiza escaladamente los cuerpos de mejor a peor. ${ }^{17}$ Esta naturalización, produce tipos ideales de cuerpos que posiciona a unos individuos como "normales", mientras estigmatiza y estereotipa a otros. La injusticia estructural más grave derivada de esos procesos de "racialización" es posiblemente, que esos procesos de "racialización" acaban determinando que "el trabajo físico, sucio y servil es más apropiado para miembros de estos grupos racializados" (Young, 2000:198-204) . Lo cual a su vez produce segregación, y determina que la gente que vive en lugares segregados, además de sufrir las consecuencias nocivas de estos lugares en términos de infraestructuras y recursos, desarrolla hábitos y comportamientos que suelen proyectarse como desviados tras la lupa distorsionante de cánones dominantes de respetabilidad y buena conducta.

Esta conclusión es importante, porque refuerza la idea de que es imposible separar injusticias culturales de aquellas otras que tienen que ver con la distribución de recursos materiales, y de que antes que polarizar injusticias estructurales en dos cate- gorías que distinguen entre economía y cultura, es más conveniente utilizar una familia de conceptos como los de explotación, estigmatización, marginalización, normalización etc. que refieran a procesos sociales que limitan oportunidades y el acceso a recursos. En su extraordinaria obra Anatomy of Racial Inequality, Glen Loury, pone de manifiesto que la estigmatización de una especificidad corporal, como lo es la del color negro, acaba generando una opresión económica como lo es la división racial del trabajo. ${ }^{18} \mathrm{~A}$ este fenómeno, se refieren algunos estudios cualitativos hechos a partir del análisis de procesos de asimilación de migrantes en algunas ciudades europeas como una incardinación en el mercado de trabajo "etnoestratificada" (Colectivo IOÉ, 2008).

Por ello, según se interpreta, es más conveniente abordar estas cuestiones desde un enfoque que presta atención a diferencias derivadas de estructuras de poder, antes que a diferencias centradas exclusivamente en características de etnicidad, nacionalidad y religión. Porque mientras que el segundo limita las cuestiones de justicia a problemas de libertad de expresión y autonomía, el primero atiende de una manera más adecuada a temas que tienen que ver con la disponibilidad o limitación sustancial de oportunidades para el desarrollo de capacidades y bienestar social. Estas relaciones categóricas de privilegio y desventaja implican que las personas situadas en posiciones subordinadas encuentran más obstáculos para perseguir sus intereses, y un margen más estrecho de oportunidades para desarrollar y ejercitar sus capacidades creativas y auto-organizativas. Cualquier conflicto multicultural, en definitiva, debería comenzar discutiendo estos temas.

\section{CONCLUSIONES}

Este artículo ha tratado de abordar la política de la diferencia desde una perspectiva crítica. No obstante, en él se refrenda una concepción determinada de la misma por entender que ésta satisface mejor los ideales de inclusión dentro de las sociedades pluralistas actuales.

\footnotetext{
${ }^{16}$ Tomo la expresión de Fanon (1967)

${ }^{17}$ Lewis Gordon analiza el racismo siguiendo esta lógica de jerarquización en GORDON (1995:29-45, 97-104) .

${ }^{18}$ En conformidad con la tesis de Glen Loury, la estigmatización de "lo negro" explica el origen de la esclavitud, y la consecuente división social-racial del trabajo en Estados Unidos.
} 
La política de la diferencia, según se muestra a lo largo del artículo, incluye un potencial emancipador grande porque supone el cuestionamiento de algunos pilares liberales fundamentales que ayudan a transformar de manera sustancial el sentido de algunas políticas públicas dirigidas a una mayor democratización en nuestras sociedades. En este artículo se han analizado concretamente dos de esos cuestionamientos. El sujeto político del liberalismo, y la concepción misma de la noción de igualdad. El objetivo con ello, ha sido el de poner de manifiesto que una teoría democrática dentro del mundo contemporáneo debe ser capaz de cruzar y abarcar las diferencias culturales y de posiciones sociales, para llegar a una igualdad sustantiva entendida como desarrollo de capacidades.

Por estos motivos, en el artículo se sugiere que, lejos de enfoques multiculturales que entienden la diferencia como identidad, la política de la diferencia más conveniente en nuestros días, debería centrarse en identificar las diferencias que surgen de estructuras de poder, antes que de diferencias centradas exclusivamente en características de etnicidad, nacionalidad y religión. Al mismo tiempo, el reconocimiento de esa diferencia como desigualdad estructural, según se interpreta, abre las puertas a un mejor entendimiento de algunos conflictos multiculturales que suelen silenciar, desplazar o banalizar otras cuestiones de justicia social, que no deberían ir desligadas en los debates sobre la diferencia cultural. Las teorías democráticas actuales necesitan en definitiva, una concepción más amplia y plural del sujeto y el objeto político, pensada sobre la base de las diferencias en relación con la posición social y la afiliación cultural de las personas.

\section{BIBLIOGRAFÍA:}

Appiah Kwame Anthony (2007) : Justice, Governance, Cosmopolitanism and the Politics of Difference: Reconfigurations in a Transnational World, Berlin, Universität zu Berlin.

Barry Brian (2001) : Culture \& Equality: An Egalitarian Critique of Multiculturalism, Harvard University Press.

Benhabib Seyla (1986) : Situating the Self, Cambridge, Polity Press.

Benhabib Seyla (1996) : Democracy and Difference, Princeton, Princeton University Press.

Benhabib Seyla (2006) : Las reinvindicaciones de la cultura, Buenos Aires, Katz Editores.

Bohman James y REGH William (1997) : Deliberative Democracy: Essays on Reason and Politics, The MIT Press.

Charles, Tilly (1998) : Durable Inequality, Berkeley, University of California Press.

COLECTIVo IOÉ (2008) : Interpretaciones de la condición migrante. Exploración de los discursos de la población inmigrada en España, CIS.

Del Águila Rafael y VALleSpín Fernando (1989) : La Democracia en sus Textos, Madrid, Alianza Editorial.

Del Águila Rafael (2008) : "Identidades culturales e integración política: un mapa conceptual”, en La Inmigración y sus causas, Editorial Sistema.

Dworkin Ronald (1981) : “What is Equality?", Philosophy and Public Affairs, 10, 1981.

Dworkin Ronald (2000) : Sovereign Virtue: The Theory and Practice of Equality, Harvard: Havard University Press.

FAnON Frantz (1967) : Black Skin, White Masks, New York, Grove.

Foucault Michel (1980) : Power/Knowledge: Selected Interviews and Other Writings, New York, Pantheon Books.

FraSER NANCY (1997a): “A rejoinder to Iris Young”, New Left Review, No 223, mayo-junio, pp. 126-129.

FRASER NANCY (1997) : Iustitia Interrupta; Reflexiones críticas desde la condición postsocialista, Universidad de los Andes.

Fraser NANCY (2008) : Scales of Justice, Columbia University Press.

Friedman Marilyn (1985) : "Harming Women as a Group", Social Theory and Practice, 11, 297-234.

Gilligan Carol (1993) : In a Different Voice, Harvard, Harvard University Press.

Gordon Lewis (1995) : Bad Faith and Anti-Black Racism, New York: Prometheus Books.

Gutmann, Amy (2004) : Identity and Democracy, Princeton University Press.

Habermas JÜrgen (1983) : Reason and the Rationalization of a Society, Boston: Beacon Press.

Hartney M. (1995) : "Some Confusions Concerning Collective Rights", en W. Kymlicka (comp.), The Right of Minoriy Cultures, Oxford: Oxford University Press.

Honneth Axel (1996) : The Struggle for Recognition: The Moral Grammar of Social Conflicts, Polity Press.

Hamlin Alan y PETTIT Philip (1989) : The God Polity: Normative Analysis of the State, London, Blackwell. 
Kelly Paul (2002) : "Defending some Dodos: Equality and/or Liberty?, en Multiculturalism Reconsidered, ed. Paul Kelly, Cambridge: Polity.

Kymlicka Will (1995), Multicultural Citizenship, Oxford: Oxford University Press.

Loury Glen (2002) : Anatomy of Racial Inequality, Cambridge, Harvard University Press.

Macintyre A. (1981) : After Virtue, Londres: Duckworh.

Nielsen K. (1985) : Equality and Liberty: A Defense of Radical Igualitarism, Roman \& Allanheld.

Nussbaum, Martha (2006) : Frontiers of Justice: Disability, Nationality, Species Membership, Harvard University Press.

Phillips, Ann (2004) . "Defending equality of outcome", Journal of Political Philosophy, 12 (1), pp. 1-19.

Rawls John (1979) : Teoría de la Justicia, México, Fondo de Cultura Económica.

Rawls, John (1993) : Political Liberalism, Columbia, Columbia University Press.

Roemer, Jonh (1998) : Equality of Opportunity, Cambridge, Cambridge University Press.

Sandel Michael (1982): Liberalism and the Limits of Justice, Cambridge, Cambridge University Press.

Sen Amartya (1982) : “Equality of What?”, en Choise, Welfare and Measurement, Oxford, Blackwell

Sen Amartya (2009) : La idea de la Justicia, Madrid: Taurus.

Spelman Elizabeth, LUGONES María (1983) : "Have We Got a Theory for You! Feminist Theory, Cultural Imperialism and the Demand for the woman's voice", Women's Studies International Forum, 6, 573-581.

Taylor Charles (1985) : “Atomism”, en Philosophical Papers, vol. 2, Cambridge: Cambridge University Press, pp. 187-210.

Vallespín, Fernando (1996) : “Introducción”, en Jürgen Habermas/ John Rawls: Debate sobre el liberalism politico, Madrid, Paidós.

Walzer Michael (1983) : Spheres of Justice, Oxford, Blackwell.

Warner Michael (1999) : The Trouble with Normal, Harvard University Press.

Wolff, Jonathan (2001) : Filosofia Politica, Una introducción, Barcelona, Ariel Filosofía.

Young Iris Marion (1990) : Justice and Politics of Difference, Oxford, Oxford University Press.

Young IrIS MARION (1996) : "Vida política y diferencia de grupo: una crítica del ideal de ciudadanía universal”, en Perspectivas feministas en Teoría Politica, Carme Castells (Comp.), Paidós.

Young Iris Marion (1997) : Intersecting Voices, Princeton, Princeton University Press.

Young Iris Marion (1997b) : “Unruly Categories: A Critique of Nancy Fraser's Dual Systems Theory”, New Left Review, no.222, March/April.

Young Iris Marion (2000) : Inclusion and Democracy, Oxford, Oxford University Press.

Young Iris Marion (2005) : On Female Body Experience, Oxford, Oxford University Press.

Young Iris Marion (2006) : "Education in the Context of Structural Injustice: A Symposium response", Educational Philosophy and Theory, Vol. 38, No. 1.

Young, Iris Marion (2007) : "Structural Injustice and the Politics of Difference" 